\title{
A Split-Based Method for Polygonal Approximation of Shape Curves
}

\author{
R. Dinesh, Santhosh S. Damle, and D.S. Guru \\ Department of Studies in Computer Science, University of Mysore, \\ Manasagangotri, Mysore - 570 006, India \\ \{dinesh_r21, emailssd\}@yahoo.com, \\ guruds@lycos.com
}

\begin{abstract}
A new split-approach based method for approximating a boundary curve by a polygon is proposed in this paper. The proposed method recursively splits boundary curve into smaller segment with the help of small eigenvalue of covariance matrix of the boundary curve. Set of boundary points at which boundary curve is split into smaller segments are considered as vertices of the approximating polygon. Experimental results show that the proposed method is robust and efficient.
\end{abstract}

Keywords: Polygonal approximation, Split approach, Eigenvalue, Integral square error, Compression ratio.

\section{Introduction}

Polygonal approximation is a simple and convenient method for shape curve representation. It offers advantages like simplicity of computation, coding efficiency, good preservation of local properties and reduction in complexity while extracting features. Moreover it offers simpler representation when compared to higher order splines and functional representation.

Several techniques have been proposed for approximating a given boundary curve by a polygon. These schemes can be broadly classified into three classes; (1) Mergebased approaches, (2) Split-based approaches and (3) Split-and-merge based approaches (Pikaz and Dinstein, 1995). Merge approaches are based on linear scanning of digital curve, where, at each point a decision is made whether to merge the point with the current straight line or to draw a new line by treating the previous point as a vertex. Split-approaches are based on recursive partitioning of a curve at points, which are farthest from a given chord connecting two points on the boundary curve. Whereas Split-and-merge based approaches make use of both merge-approach and split-approach for polygonal approximation.

Ray and Ray (1992) proposed a k-cosine based algorithm for polygonal approximation of shape curves. This method makes use of k-cosine for determination of asymmetric region of support and identifies dominant points on the boundary, which are considered as the vertices of approximating polygon. Though the method produces fairly good approximation, it is computationally inefficient as it involves huge trigonometric calculations. Wang et al., (1995) proposed a computationally 
efficient method, which uses bending value to detect corner points on a curve. However, the method fails to detect sharp corner points, which results in poor approximation. A method proposed by Pikaz and Dinstein (1995) for polygonal approximation builds a heap data structure with the contour points and recursively approximates the curve. Nevertheless the method requires two error criteria to be chosen by the user. Tsai et al., (1999) proposed a method for detection of corner points based on small eigenvalue. The result of this method is very sensitive to the input parameter and in fact it is very difficult to choose a parameter, which effectively works for all kinds of shapes. Methods based on dynamic programming (Perez and Vidal (1994), Marc Salotti (2002) Kolesnikov and Franti(2003)) were also proposed for approximating a boundary curve by a polygon. The main drawback of the methods based on dynamic programming is that, they are computationally very expensive as they include exhaustive searching while identifying the vertices of the approximating polygon and hence they are of little use in real pragmatic applications. Some other interesting workds could be Ray and Ray (1995), Zhang and Guo (2001), Marji and Siy (2003) and Guru and Dinesh (2004).

From the above discussion it is clear that the existing schemes for approximating a shape by a polygon exhibit some shortcomings. The methods that are effective are not computationally efficient and the methods that are computationally efficient suffer from poor approximation. In view of this, in this paper, a successful attempt is made to device a scheme for polygonal approximation. The proposed approach in addition to being computationally efficient, produce acceptable quality results. The proposed method is based on split-approach, it splits the boundary recursively at points which would act as vertices of approximating polygon. Small eigenvalue of covariance matrix of the boundary segment is used as stopping criterion.

\section{Proposed Method}

The proposed method uses Split-approach to detect vertices of a polygon approximating a boundary curve. Boundary curve is recursively split into smaller segments. During each split, a decision is taken as to go for further splitting or not and the decision is based on the small eigenvalue $(\lambda)$ of covariance matrix of boundary points. The points at which the boundary curve is split into smaller segments are considered as the vertices of the approximating polygon.

For a set of points $\mathrm{S}=\left\{\mathrm{P}_{\mathrm{i}=}\left(\mathrm{x}_{\mathrm{i}}, \mathrm{y}_{\mathrm{i}}\right) \mid \mathrm{i}=1,2, \ldots, \mathrm{n}\right\}$ small eigenvalue $\lambda$ is computed as:

$$
\lambda=\frac{1}{2}\left[c_{11}+c_{22}-\sqrt{\left(c_{11}-c_{22}\right)^{2}+4 c_{12}^{2}}\right]
$$

where, $\quad\left[\begin{array}{ll}c_{11} & c_{12} \\ c_{21} & c_{22}\end{array}\right]$

is the covariance matrix of $\mathrm{S}$, and the coefficients $\mathrm{C}_{11}, \mathrm{C}_{12}, \mathrm{C}_{21}$ and $\mathrm{C}_{22}$ are calculated as below: 


$$
\begin{aligned}
\mathrm{C}_{11} & =\frac{1}{n} \sum_{i=1 \ldots n} x_{i}^{2}-c_{x}^{2} \\
\mathrm{C}_{12} & =\mathrm{C}_{21}=\frac{1}{n} \sum_{i=1 \ldots n} x_{i} \cdot y_{i}-c_{x} \cdot c_{y} \\
\mathrm{C}_{22} & =\frac{1}{n} \sum_{i=1 \ldots n} x_{i}^{2}-c_{x}^{2}
\end{aligned}
$$

Here, $c_{x}=\frac{1}{n} \sum_{i=1 \cdots n} x_{i}$ and $c_{y}=\frac{1}{n} \sum_{i=1 \cdots n} y_{i}$ are the respective mean values of the $\mathrm{x}$ and $\mathrm{y}$ coordinates of the points in $\mathrm{S}$.

Theoretically straight lines have associated small eigenvalue $(\lambda)$ equal to zero and digital lines have their $\lambda$ value closer to zero. Proposed method uses this statistical property for determining whether a boundary segment needs further splitting or not. If the boundary segment is approximately a straight line then it does not require further splitting.

Let $\mathrm{B}$ be a closed boundary with $\mathrm{n}$ points, i.e., $\mathrm{B}=\left\{\mathrm{P}_{\mathrm{i}}=\left(\mathrm{x}_{\mathrm{i}}, \mathrm{y}_{\mathrm{i}}\right), \mathrm{i}=1,2,3 \ldots, \mathrm{n}\right\}$, where points $\mathrm{P}_{\mathrm{i}}$ and $\mathrm{P}_{\mathrm{i}+1}$ (modulo $\mathrm{n}$ ) as neighbors.

The proposed method, initially identifies two farthest points $\mathrm{P}_{\mathrm{q}}$ and $\mathrm{P}_{\mathrm{r}}$ on the boundary curve $B$. This splits the boundary curve into two segments $S_{q, r}=\left\{P_{k} \mid k=q\right.$ to $\mathrm{r}$ (modulo $\mathrm{n}$ ) $\}$ and $\mathrm{S}_{\mathrm{r}, \mathrm{q}}=\left\{\mathrm{P}_{\mathrm{k}} \mid \mathrm{k}=\mathrm{r}\right.$ to $\mathrm{q}$ (modulo $\mathrm{n}$ ) $\}$. Both the segments are subjected as input to a recursive procedure one by one to locate the vertices of the approximating polygon. We specifically choose farthest points on the boundary curve for initial splitting to make the procedure invariant to starting point and generally two such farthest points are corner points.

Recursive procedure takes a segment $S_{x, x+y}$ at a time for processing. First it marks both end points $\mathrm{P}_{\mathrm{x}}$ and $\mathrm{P}_{\mathrm{x}+\mathrm{y}}$ as vertices of approximating polygon. Small eigenvalue for the segment is calculated; if the computed small eigenvalue exceeds predefined threshold value $\lambda_{t}$, then the segment needs further splitting, as the segment is not a straight line. Otherwise the segment does not require further processing, because the segment is roughly a straight line. To determine the split point $P_{s}$ between $P_{x}$ and $P_{x+y}$ the procedure calculates distances from each point on the segment to the chord joining $P_{x}$ and $P_{x+y}$. Point $P_{s}$ on the segment, which has a maximum distance to the chord joining $\mathrm{P}_{\mathrm{x}}$ and $\mathrm{P}_{\mathrm{x}+\mathrm{y}}$, is selected as the splitting point. Now the procedure has ended up with two curve segments $S_{x, s}$ and $S_{s, x+y}$, which would be processed by the same procedure one after the other. When this procedure ends, a set of segments are obtained, none of which have their $\lambda$ value exceeding the threshold value $\lambda_{t}$. End points of these segments are considered as the vertices of approximating polygon, and polygonal approximation of the shape curve is obtained by joining successive vertices by a straight line. The proposed method ensures good approximation as boundary points between any pair of successive vertices form a straight line.

Optional post processing stage is also proposed in this work to further reduce error to improve the compression ratio. Let us assume that $\mathrm{m}$ is the number of vertices obtained by the above split procedure i.e., $\mathrm{V}_{\mathrm{i}}, \mathrm{i}=1$ to $\mathrm{m}$. Square Error between pair of points $\mathrm{j}$ and $\mathrm{k}$ are denoted as $\mathrm{E}_{\mathrm{j}, \mathrm{k}}$. 
Following are the optional post processing rules suggested:

Rule 1: Rule to reduce overall error

Some of the candidate vertices obtained by split procedure may not be the exact vertices. In such cases, exact vertex would be in the vicinity of obtained vertex. Overall error would reduce, if exact vertices are identified and vertices obtained by split procedure are replaced by them. Procedure for identification of exact vertices is explained as follows:

Let $\mathrm{E}_{\mathrm{i}}$ be sum of square errors between segments $\left(\mathrm{V}_{\mathrm{i}}, \mathrm{V}_{\mathrm{i}+1}\right)$ and $\left(\mathrm{V}_{\mathrm{i}}, \mathrm{V}_{\mathrm{i}-1}\right)$. Shift the vertex $V_{i}$ to its neighboring points $P_{j}(j=-2 . .+2)$ and calculate error $E_{j}$ in each case. Select the point $P_{j}$ as actual vertex for which the computed error $E_{j}(j=-2 . .+2)$ is least. This reduces the error between successive vertices and thus overall error due to approximation is also reduced, and hence approximation is improved.

\section{Rule 2: Rule to improve compression ratio}

If there are any $\mathrm{V}_{\mathrm{i}} \mathrm{s}$ on straight line or on a curve segment with negligible curvature formed by its neighboring vertices $V_{i-1}$ and $V_{i+1}$ (modulo $m$ ) such point can be eliminated. For each set of vertices $\mathrm{V}_{\mathrm{i}-1}, \mathrm{~V}_{\mathrm{i}}, \mathrm{V}_{\mathrm{i}+1}$, calculate the distance between $\mathrm{V}_{\mathrm{i}}$ and the chord joining $\mathrm{V}_{\mathrm{i}-1}$ and $\mathrm{V}_{\mathrm{i}+1}$. If this distance is less than a predefined threshold $\mathrm{D}_{\mathrm{t}}$, then we recommend it to be eliminated. So the number of vertices $\mathrm{m}$ is reduced to $\mathrm{m}-1$. This process is an iterative procedure, each iteration works for all the vertices obtained from split procedure or from previous iteration. Iterative procedure is terminated when no vertex is eliminated during an iterative loop. This rule helps us in improving the compression ratio keeping the error unchanged.

Therefore, algorithm for the proposed method of approximating a given shape by a polygon is as trivial as follows.
Algorithm: Polygon approximation.
Input: An object / shape.
Output: Corresponding polygonal approximation of the input object/shape.
Method:
1. Extract boundary $(B)$ of input image.
2. Select two farthest points on the image boundary; let them be $P_{i}$ and $P_{j}$.
3. Recursively split segments $\left(P_{i+1}, P_{j}\right)$ and $\left(P_{j+1}, P_{i}\right)$ as explained above.
4. Apply optional post-processing rules to further improve the quality of approximation.

\section{Algorithm ends.}

\section{Experiments}

In order to establish the superiority of the proposed algorithm several experiments were conducted on various standard shapes/boundaries, which also included shapes considered by other researchers. Out of various shapes considered for experimentation, a few shapes and the obtained results are given in Table 1. Through out the experimentation, values of the parameters $\lambda_{t}$ and $D_{t}$ were set to 0.25 and 1.1 respectively. 
Table 1. Some of the test shapes on which proposed algorithm was applied and their results

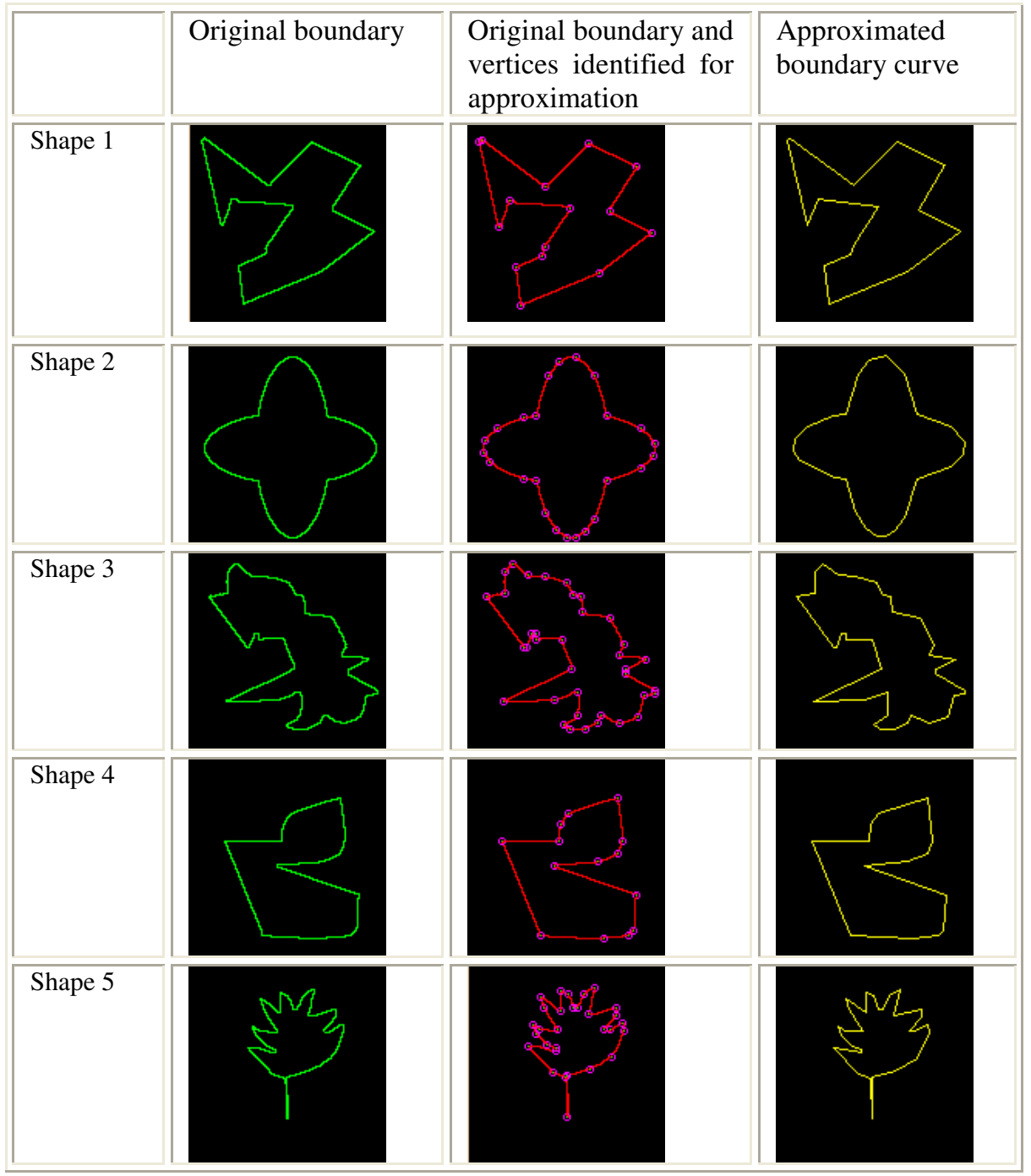

Table 2. Compression ratio and error of approximation for the shapes considered in Table 1

\begin{tabular}{|l|l|l|l|l|}
\hline & $\mathrm{N}$ & $\mathrm{V}$ & $\mathrm{CR}$ & $\mathrm{ISE}$ \\
\hline Shape1 & 638 & 15 & 42.53 & 25.24 \\
\hline Shape2 & 456 & 24 & 19 & 23.71 \\
\hline Shape3 & 622 & 37 & 16.81 & 12.02 \\
\hline Shape4 & 444 & 14 & 31.71 & 5.39 \\
\hline Shape5 & 441 & 30 & 14.7 & 10.30 \\
\hline
\end{tabular}


In order to do a quantitative evaluation of the proposed method, Integral Square Error (ISE) and compression ratio (CR) were used as measures for evaluation (Rosin 1997). Table-2 summarizes the ISE and CR due to application of the proposed methodology on the shapes shown in Table-2, along with the number of boundary points $(\mathrm{N})$ and the number of vertices $(\mathrm{V})$ identified for polygonal approximation. Both optional rules were applied to improve the Integral Square Error and Compression Ratio.

\section{Summary and Conclusions}

A new method for approximating a boundary curve by a polygon has been proposed in this work. The proposed method is based on split-approach. It detects vertices of approximating polygon by recursively splitting the boundary curve into smaller segments and uses small eigenvalue of covariance matrix of the segment as stopping criterion for splitting. Method has produced good results in terms of approximation error and compression ratio for the images used in experimentation.

\section{References}

1. Alexander Kolesnikov and Pasi Franti - 2003, Reduced-search dynamic programming for approximation of polygonal curves, Pattern Recognition Letters 24 (2003) 2243-2254.

2. Guru D.S. and Dinesh R. - 2004, Non-parametric adaptive region of support useful for corner detection: a novel approach, Pattern Recognition 37(2004) 165-168.

3. Juan-Carlos Perez and Enrique vidal - 1994, Optimum polygonal approximation of digitized curves, Pattern Recognition Letters 15(1994) 743-750.

4. Majed Marji and Pepe Siy - 2003, A new algorithm for dominant points detection and polygonization of digital curves, Pattern Recognition 36(2003) 2239-2251.

5. Marc Salotti - 2002, Optimal polygonal approximation of digitized curves using the sum of square deviation criterion, Pattern Recognition 35 (2002) 435-443.

6. Pikaz A. and Dinstein I. - 1995, an algorithm for polygonal approximation based on iterative point elimination, Pattern Recognition Letter 16(1995) 557-564.

7. Ray B.K. and Ray K.S. - 1992, an algorithm for detection of dominant and polygonal approximation of digitized curves, Pattern Recognition Letters 13(1992) 849-856.

8. Ray B.K. and Ray K.S. - 1995, a new split-and-merge technique for polygonal approximation of chain coded curves, Pattern Recognition Letters 16(1995) 161-169.

9. Rosin P.L., Techniques for Assessing Polygonal Approximation of curves, IEEE Transaction on Pattern Analysis and Machine Intelligence, Vol. 19, No. 6, June 1997.

10. Tsai D.M., Hou H.T., Su H.J. - 1999, Boundary-based corner detection using eigenvalue of covariance matrices, Pattern Recognition Letters 20(1999) 31-40.

11. Wang M.J.J., Wu W.Y., Huang L.K. and Wang D.M. - 1995, Corner detection using bending value, Pattern Recognition Letters 16(1995) 575-583. 